Musées, Patrimoine et Culture scientifiques et techniques

$117 \mid 2008$

mai - juin 2008

\title{
Photographie, musée et pouvoir : formes, ressorts et perspectives
}

\section{Elsa Olu}

\section{OpenEdition \\ Journals}

Édition électronique

URL : http://journals.openedition.org/ocim/326

DOI : 10.4000/ocim.326

ISSN : 2108-646X

Éditeur

OCIM

Édition imprimée

Date de publication : 1 mai 2008

Pagination : 14-18

ISSN : 0994-1908

\section{Référence électronique}

Elsa Olu, «Photographie, musée et pouvoir : formes, ressorts et perspectives », La Lettre de l'OCIM [En ligne], 117 | 2008, mis en ligne le 01 décembre 2009, consulté le 19 avril 2019. URL : http:// journals.openedition.org/ocim/326 ; DOI : 10.4000/ocim.326 
faits (la photographie comme «preuve » de visite à destination de touristes en mal de représentation d'eux-mêmes, un support de mémoire, fort utile dans une société où la carte flash s'apparente chaque jour davantage à une part de cerveau externalisée), ils défendent également l'acte photographique comme outil au service d'un musée singulier que la possession de photographies permettrait à chaque individu de constituer : en écho au Musée imaginaire de Malraux, c'est bien la possibilité de «fabriquer son propre Louvre » que confère désormais à chacun l'acte photographique.

Argumentaire de bonne tenue donc, qui vient s'ajouter à une litanie déjà bien longue, et qui a l'avantage de se poster non plus seulement du côté des pratiquants (les visiteurs photographes) mais du côté de l'institution elle-même, en prenant le parti non de l'acculer mais de vanter les « avantages » que lui confèrerait la « légalisation » et l'instrumentation d'une pratique qu'elle s'obstine à considérer comme potentiellement dangereuse.

Mais ce déplacement de l'argument peut-il suffire à une levée des interdits ? Plutôt que de compléter la kyrielle d'arguments pour la pratique, il n'est pas inintéressant de s'interroger sur les motivations réelles d'une institution qui s'obstine visiblement à l'interdire. Les faux prétextes du droit d'image ou du droit d'auteur écartés (Serge Chaumier et Véronique Parisot s'en chargent notamment fort bien), qu'en est-il de ces résistances et de leurs ressorts, comme des motivations avouées ou inavouables de l'institution? Le retournement des principaux arguments pour la photographie, du point de vue de l'institution muséale et en regard de ses prérogatives et de ses pouvoirs actuels, permet l'analyse des conséquences «négatives » de la pratique photographique et fournit quelques éclaircissements...

\section{L'envers du décor}

L'acte photographique qui permet l'effectuation individuelle du Musée imaginaire est également un "rapt " qui opère un déplacement de la propriété de l'objet.

Si la photographie peut permettre à chacun de «fabriquer son propre Louvre », la « mise en boîte » de l'objet peut tout autant apparaître comme un dispositif d'appropriation offert au visiteur, par lequel il lui est permit de «privatiser » l'objet, c'est-à-dire aussi de " dé-privatiser » l'objet jusque-là pleine propriété du musée. On est ici du côté du « rapt », des tactiques et des stratégies de Michel de Certeau, dans la droite ligne des Arts de faire et de ses invites à une pratique «délinquante » du musée ${ }^{(2)}$. Converti en termes juridiques, cela signifie que le peuple est - à nouveau ou enfin ? - nu-propriétaire de l'œuvre, et que l'institution n'est qu'usufruitière. À l'heure où l'objet dématérialisé (l'image numérique) a acquis non seulement une fonction mais une valeur, où le principe d'inaliénabilité des collections est remis en cause, l'institution peut appréhender l'acte photographique non plus seulement comme un «rapt » symbolique, mais comme réelle dépossession, atteinte à son intégrité, à son essence même (la collection) et à sa fonction (être l'unique conservatoire des objets).

L'acte photographique qui met en lien le visiteur et l'objet opère une médiation directe qui exclut l'institution et efface le corps physique du musée.

$\mathrm{Si}$ on peut penser la photographie comme dispositif permettant de maintenir pérenne le lien au musée "C'est pour l'individu autre chose qu'une mise en boîte, c'est une assurance sur son propre devenir, c'est aussi un lien indéfectible tissé avec un musée, une institution » argumentent Serge Chaumier et Véronique Parisot -, il est aussi possible de considérer que c'est davantage le lien à l'objet, à l'œuvre, au discours, à l'histoire, qui est "mis en image ", avec pour conséquence, non l'activation ou le renforcement d'un lien à l'institution, à la structure musée, mais, précisément, son délitement.

Envisagé ainsi, l'acte photographique apparaît bien comme une mise en lien effective du visiteur à l'objet, et la photographie un outil efficace de médiation, mais qui, au contraire de structurer temporellement et spatialement le lien du visiteur au musée, conduit à la dématérialisation de la «structure-musée », à son

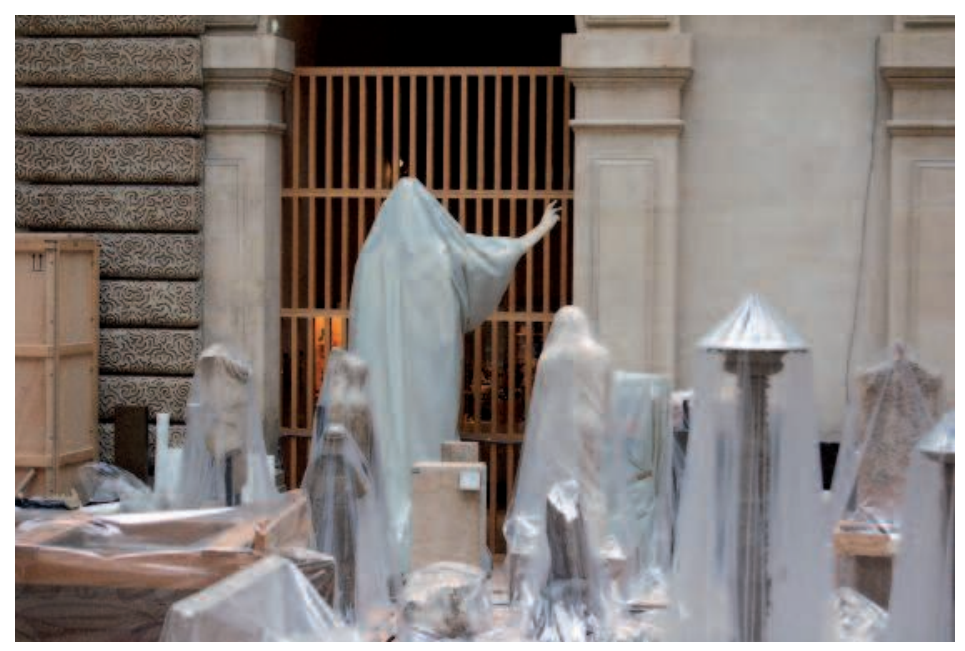

Musée du Louvre, Paris, 2008 (C) Louise Merzeau 
effacement. À la photographie entendue comme matérialisation du lien du visiteur à l'objet de musée répond l'effacement du pouvoir de "donner à voir », qui conduit à dissocier le lieu physique (le musée) du pouvoir symbolique (de l'institution), le second étant dès lors, du point de vue de l'institution, diminué du premier.

L'acte photographique qui commue le visiteur en médiateur retire à l'institution l'apanage de la transmission.

$\mathrm{Si}$, comme le développent Serge Chaumier et Véronique Parisot, le visiteur, par la photographie qu'il prend, assure lui-même un acte de médiation, et devient l'auto-médiateur du savoir, il destitue par-là même le musée d'une fonction de transmission qu'il était jusque-là seul à détenir. Autant dire, autoriser le visiteur à accomplir l'acte photographique, c'est du point de vue de l'institution légitimer une pratique qui institue le visiteur comme médiateur et accepter de partager sa mission de transmission.

Bien entendu, on s'accorde depuis quelques décennies sur le fait que désormais le musée n'a plus l'apanage ni de la communication, ni de la transmission. L'essor des TIC a profondément modifié les conditions d'accès à l'information, de communication des contenus, tout autant les processus cognitifs et les modalités de production du savoir. Si le développement du Web 2.0 et la technologie wiki ont signé la dernière mise en œuvre de "l'intelligence collective » (Pierre Lévy) en rendant l'individu acteur de l'édification collaborative des savoirs (Alain Ehrenberg) et producteur effectif de ces derniers, l'évolution des techniques et des pratiques photographiques (des outils comme des modalités de prise de vue) place désormais le visiteur de musée au cœur même de la médiation, comme visiteur-acteur du musée.

Dans le cadre d'une "nouvelle écologie cognitive » (Pierre Lévy), cette évolution, inéluctable, d’un visiteur prétendument passif vers un visiteur ouvertement actif, ne peut qu'être envisagée par tout un chacun comme positive. Il n'en reste pas moins que, du point de vue d'une institution encore à bien des égards repliée sur ses archaïsmes et avant tout intéressée par le maintien de ses prérogatives, les choses ne sont pas si aisées... Pour considérer l'argument exposé par Serge Chaumier et Véronique Parisot, il lui faudrait s'attacher au résultat de la pratique (l'optimisation du processus de médiation des contenus et des savoirs) quand elle ne retient, elle, que le déplacement des rôles et la perte effective du pouvoir corrélé.
L'acte photographique qui octroie au visiteur un droit de présentation et de représentation de l'objet dépossède l'institution d'un efficace dispositif d'autoreprésentation.

Enfin, il apparaît que céder pour partie la propriété de l'œuvre (l'image) en autorisant que sa présentation puisse être assurée par un tiers non-institutionnel dans un espace non maitrisé (notamment virtuel), retire à l'institution le privilège du métadiscours qui, non seulement édicte la façon dont l'objet présenté doit être regardé (cadrage de la médiation), mais prive également l'institution d'un précieux dispositif d'autoreprésentation : si l'on considère que le processus d'exposition est un dispositif autoréférentiel par lequel l'institution se présente, se dit et s'impose, l'acte photographique apparaît de toute évidence comme un déstabilisateur non négligeable...

C'est donc bien plus que la qualité d'agent de médiation de la photographie que les deux auteurs, Serge Chaumier et Véronique Parisot, mettent en exergue. L'acte photographique est un acte muséologique et muséographique par excellence, qui commue le visiteur en acteur du musée : par lui, il devient possiblement collectionneur (qui tisse un lien affectif à l'œuvre), conservateur (il archive sur sa carte flash), propriétaire (il fait «son » musée), commissaire d'exposition (il organise ses dossiers, rapproche les œuvres de clics en clics selon son discours propre...), (auto)-médiateur (il développe un discours explicatif, adjoint des commentaires écrits captés au fil du Web ou des impressions enregistrées, assure leur transmission à des tiers via sites participatifs, blogs, forums, chats...)...

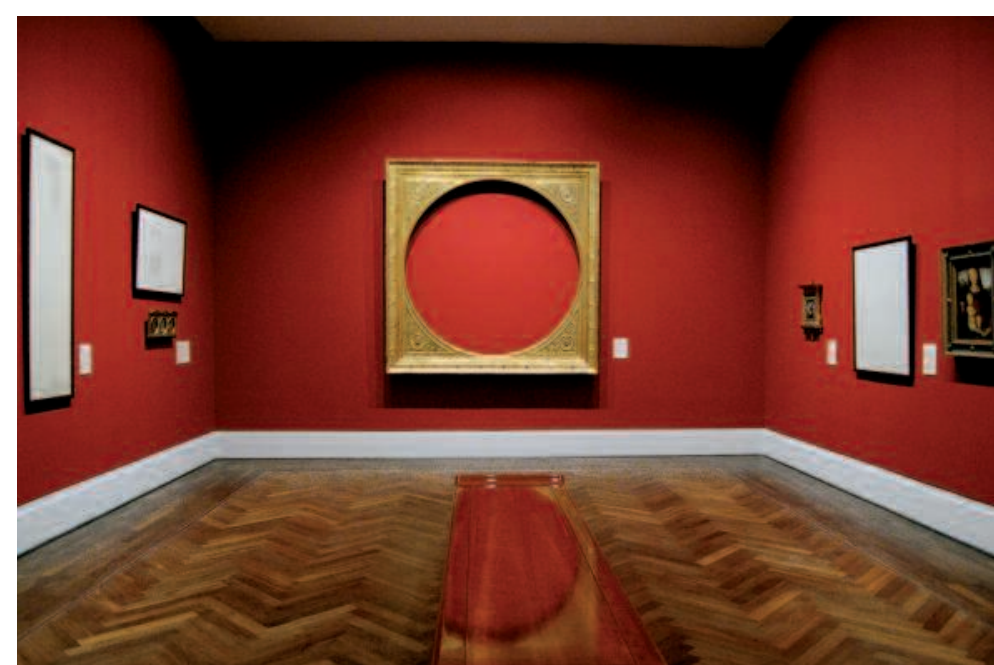

Gemäldegalerie, Berlin, 2007 (c) Louise Merzeau 
L’argument ainsi retourné laisse apparaître les craintes probables d'une institution d'être dépossédée de ce qui la constitue : la collection (dématérialisée et emportée par le visiteur), la structure physique de représentation de celle-ci (le musée), la fonction même de la «structure-musée » dans l'espace public (le musée n'est plus indispensable dans la relation œuvre-visiteur), la responsabilité de la médiation (qu'elle veillait à cadrer, encadrer, maîtriser) et l'apanage de la transmission.

Plus encore, c'est sans doute du risque de se voir privée du dispositif même par lequel elle s'autoreprésente, partant, s'autolégitime, que l'institution semble bien prendre toute la mesure. Car en autorisant l'acte photographique, c'est bien toute une " microphysique du pouvoir » qui est mise à l'épreuve...

\section{Actualités foucaldiennes}

L'acte photographique au musée : une microphysique du pouvoir à l'épreuve

Une relecture de Michel Foucault permet d'envisager le musée comme une véritable «technologie de pouvoir » : l'organisation du musée est le cadre de mise en œuvre d'une "microphysique du pouvoir ", qui passe par l'édiction et le maintien d'une discipline dont l'acte transgressif apparaît durement réprimable (3). La réglementation des pratiques, la pression exercée sur des corps rendus dociles (ne touchant pas, ne disant mot, silencieux et calmes, le regard haut, les mains derrière le dos...), définissent non seulement les conditions de pratique de l'espace physique du musée mais l'animent de dynamiques qui confèrent autant à l'institution de « micro pouvoirs » qu'elles les signifient ou les rappellent à tout un chacun.

Or, comme permet de le conclure le retournement des arguments précédents, c'est précisément cette microphysique du pouvoir que la prise de vue vient bousculer et à laquelle l'autorisation de photographier porte atteinte.

L'interdit photographique ne fait donc jamais que s'inscrire dans la continuité des interdits auxquels le visiteur est bien accoutumé, et que l'institution maintient comme dispositif opérant de soumission de l'individu à une pratique sacralisée de l'église-muséale, empreinte d'une double morale, platonicienne et chrétienne. Dans la fabrique du corps pratiquant, le musée s'avère être un dispositif depuis longtemps opérant, et autoriser l'acte photographique conduirait ni plus ni moins qu'à la fragilisation du dispositif disciplinaire qu'est l'espace muséal, avec pour

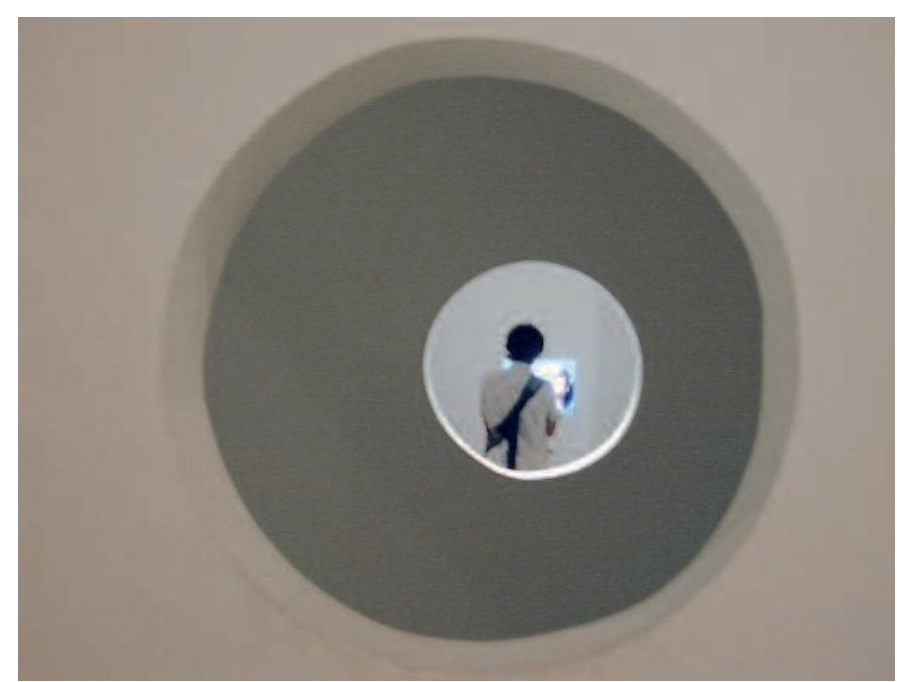

Exposition Alfred Hitchcock et l'art, Centre Georges Pompidou, Paris, 2001

(c) Louise Merzeau

conséquence le démantèlement du musée comme espace symbolique de structuration des rôles (entre l'Institution et le Peuple) la désorganisation dans l'organisation des pouvoirs et la perte (de pouvoir et de légitimité) afférente ${ }^{(4)}$.

En adoptant ce point de vue, on comprend davantage la position de l'institution muséale, et l'usage qu'elle fait des prétextes les plus fallacieux pour maintenir l'interdit photographique : en s'arc-boutant sur des logiques de propriété, en revendiquant des droits à l'image ou en s'abritant derrière des prétextes de conservation, l'institution ne fait jamais que préserver l'équilibre des pouvoirs au sein même de son espace comme garantie, croit-elle, de sa légitimité dans l'espace public.

Face à ces risques, l'instauration d'une nouvelle "Liberté surveillée »

Cependant, la réalité n'étant pas si tranchée et caricaturale, il faut évoquer les démarches récentes de certains musées qui, acculés par les pressions des visiteurs et des professionnels, tentent d'aménager l'interdit plus que l'autorisation de la pratique, et prennent le parti de «feindre d'autoriser » en proposant une forme originale de « liberté surveillée ».

L'exemple le plus parlant, rapporté par Serge Chaumier et Véronique Parisot, est la mise en place, lorsque l'institution autorise la prise de vue dans l'espace muséal, de panneaux ou dispositifs visant à indiquer aux visiteurs le meilleur angle de prise de vue pour prendre leur photographie. La présence de tels points d'information aux fonctions prescriptives apparait à l'évidence comme une déclinaison nécessaire de la «microphysique du pouvoir » : en déterminant les 
postures du corps (le corps photographiant et le corps photographié), l'institution opère le renouvellement de ses techniques et moyens disciplinaires au service de la gouvernance des corps. Ce qui pourrait apparaitre comme une ouverture de l'institution à une pratique individuelle et libre des visiteurs n'est jamais qu'un nouveau dispositif de contrôle de l'institution qui atteste d'une véritable capacité à adapter sa microphysique du pouvoir aux évolutions des pratiques sociales. Geneviève Vidal fait une analyse similaire en s'attachant à l'usage que font les institutions de leurs sites Internet ${ }^{(5)}$. On peut tout autant y voir, dans une lecture foucaldienne, le développement d'un nouveau dispositif de contrôle, de déploiement de stratégies disciplinaires servant au maintien de leur pouvoir sur les individus.

\section{Vers I'ère post-muséale}

Lacte photographique est donc vécu par l'institution comme autant de tentatives de déstabilisation d'une microphysique du pouvoir qu'elle veille depuis toujours à maintenir, et qui vacille chaque jour davantage sous le joug des avancées technologiques : les technologies et les nouvelles pratiques auxquelles elles donnent lieu modifient en effet non seulement les pratiques dans l'espace public mais tout autant les pratiques cognitives et culturelles. Leur impact sur les « institutions légitimes » du savoir et sur le musée en particulier est évident, et si l'institution entend dessiner au musée un avenir, il lui faut désormais envisager celui-ci en regard des nouvelles configurations sociales.

Ouvrir la voie d'une « démodynamique » culturelle (au sens entendu par Lévy, du grec dunamis : force, puissance) et engager une double mobilisation, subjective individuelle d'une part, éthique et coopérative d'autre

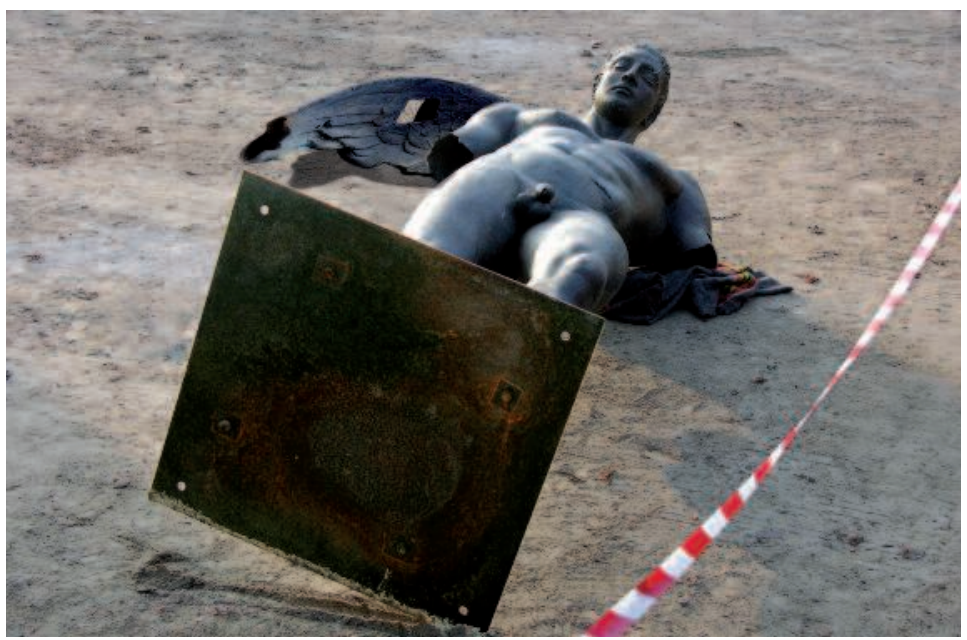

Sculpture d'Igor Mitoraj, jardin des Tuileries, Paris, 2004 (c) Louise Merzeau part : ces deux voies sont conditionnées par la capacité de l'institution à accepter le démantèlement de cette microphysique du pouvoir et suppose le fondement d'une néo-muséologie (6).

Dès lors, un nouveau paradigme muséal peut émerger, qui propose une hybridation pertinente des territoires de pratiques et de savoirs (espace public, Web et institutions), qui, en miroir du Web 2.0, de ses logiques non-linéaires et de ses nouvelles formes de sociabilité, soit en mesure d'ouvrir sur une nouvelle ontologie culturelle. Car de Barthes il faut aussi retenir «mon fantasme, l'idiorythmie » (7), puis revenir sur cette idée « de certienne » : traficoter avec le musée pour éprouver le monde. Entre le musée mortifère du siècle dernier tout emmailloté de verre où il n'est rien permis de vivre, et l'agora faussement bâtie pour une appartenance commune de circonstance, il y a assurément dans l'espace public la place d'une forme médiane, mouvante et devenante, offerte à tous nos petits trafics.

Les technologies auxquelles appartient la photographie doivent être envisagées non comme des menaces mais comme les clefs d'entrée dans l'ère post-muséale (8), les nouvelles pratiques culturelles, technologiques et cognitives, comme les outils salutaires qui substitueront à l'utopie de la démocratisation culturelle l'ébauche d'une démocratie culturelle. Il est temps de faire entrer le monde dans les salles sombres, d'animer le musée de nos réalités et de nos vitalités, de bousculer ses cadres pour le réinventer. Stiegler disait « La question qui se pose aujourd'hui est : comment articule-t-on la technique avec les choses sublimes ?». Il est aujourd'hui temps d'essayer, en engageant une véritable transvaluation de l'ensemble du système, au sens nietzschéen du terme, et en entrant dans l'ère post-muséale.

\section{Notes}

(1) Chaumier, S. et Parisot, V. Un nouvel interdit au musée : la photographie ?, la Lettre de l'OCIM n 115 , janvier-février 2008, pp. 23-30. (2) Certeau (de) M. L'invention du quotidien-Arts de faire. Paris Gallimard, 1990.

(3) Foucault, M. Surveiller et punir. Paris : Gallimard, 1975 et pour l'usage spécifique de Foucault dans l'approche des dispositifs d'information et de communication : Bertini, M.-J. Communication et Technologies du Pouvoir, Synthèse des travaux d'Habilitation à Diriger des Recherches, université Stendhal-Grenoble 3, 2005.

(4) Olu, E. Ouvrir l'ère post-muséale. Propositions pour une néo-muséologie au service d'une nouvelle ontologie culturelle, Thèse de doctorat sous la direction de Bernard Deloche, université Jean-Moulin Lyon III, à paraître. (5) Vidal, G. Contribution à l'éloge de l'interactivité : les usages du multimédia de musée. Bordeaux : Presses universitaires de Bordeaux, 2006. (6) Olu, E., op. cit.

(7) Barthes, R. Mon fantasme, l'idiorythmie. Comment vivre ensemble (cours et séminaires au Collège de France 1976-1977). Paris Seuil/IMEC, 2002.

(8) Olu, E., op. cit. 